\title{
Computing the Stopping Distance of a Tanner Graph is NP-hard
}

\author{
K. Murali Krishnan and Priti Shankar
}

\begin{abstract}
Two decision problems related to the computation of stopping sets in Tanner graphs are shown to be NP-complete. It follows as a consequence that there exists no polynomial time algorithm for computing the stopping distance of a Tanner graph unless $\mathbf{P}=\mathbf{N P}$.
\end{abstract}

Index Terms - Tanner graph, stopping distance, NP-hardness, low-density parity-check (LDPC) codes.

\section{INTRODUCTION}

Stopping sets were introduced in [1] for the analysis of erasure decoding of low-density parity-check (LDPC) codes. It was shown that the iterative decoder fails to decode to a codeword if and only if the set of erasure positions is a superset of some stopping set in the Tanner graph [8] used in decoding. Considerable analysis has been carried out on the distribution of stopping set sizes in LDPC code ensembles, giving valuable insight into the asymptotic performance of message-passing decoding on LDPC ensembles — see for example [2], [3]. Since small stopping sets are directly responsible for poor performance of iterative decoding algorithms, it is of interest to determine the size of the smallest stopping set in a Tanner graph, called the stopping distance of the graph. Construction of codes for which there are Tanner graphs that do not contain small stopping sets has been studied — see for example [4], [5]. The stopping distance of the graph, is of interest as it gives the minimum number of erasures that can cause iterative decoding to fail.

The relationship between stopping distance and other graph parameters like girth has been explored in [6] where it is shown that large girth implies high stopping distance. PishroNik and Fekri [12] showed that by adding a suitable number of parity checks the stopping distance of a Tanner graph for a code can be increased to the maximum possible, viz., the minimum distance of the code. Schwartz and Vardy [7] define the stopping redundancy of a code as the minimum number of rows in a parity check matrix for the code, such that the stopping distance of the corresponding Tanner graph is equal to the minimum distance of the code, and prove some bounds on the stopping redundancy for various classes of codes. Further investigations on stopping redundancy may be found in [13]. 2006.

A preliminary version of this paper was presented at CISS 2006, March

K. Murali Krishnan acknowledges sponsorship for PhD.from the National Institute of Technology, Calicut under the QIP scheme.

The work of the authors is supported by the Defence Research and Development Organization, India, under grant DRDO/ECA/PRS/590. The authors are with the Department of Computer Science and Automation, Indian Institute of Science, Bangalore, India. e-mail: \{kmurali,priti\}@csa.iisc.ernet.in
In this correspondence, we show that the computational problems of determining whether a given Tanner graph has a stopping set of a given size or of at most a given size are NPcomplete. These are shown, respectively, by reductions from the well known NP-complete problems of determining whether a given graph contains a vertex cover of a given size or of at most a given size. As a consequence of the latter result, it follows that there exists no polynomial time algorithm for the problem of computing the stopping distance of a Tanner graph unless $\mathrm{P}=\mathrm{NP}$.

\section{BACKGROUND}

Given a parity check matrix $H=\left[h_{i j}\right] \in G F(2)^{(n-k) \times n}$, $1 \leq k \leq n$ for an $(n, k)$ binary linear code, the Tanner graph is the undirected bipartite graph $G=(L, R, E)$ where $L=$ $\left\{x_{i}, 1 \leq i \leq n\right\}, R=\left\{c_{j}, 1 \leq j \leq n-k\right\}$ and $E=\left\{\left(x_{i}, c_{j}\right)\right.$ : $\left.h_{j i}=1,1 \leq i \leq n, 1 \leq j \leq n-k\right\}$. The set $L$ corresponds to the set of codeword elements and $R$ corresponds to the set of parity checks. We refer to the set $L$ and $R$ as the set of left and right vertices respectively. Note that as $G$ is undirected, $E$ consists of unordered pairs and thus both $\left(x_{i}, c_{j}\right)$ and $\left(c_{j}, x_{i}\right)$ refer to the same edge in the graph for all $1 \leq i \leq n, 1 \leq j \leq$ $n-k$. For $S \subseteq L \cup R$, we define $N(S)=\{y:(x, y) \in E, x \in$ $S\} . S \subseteq L$ is a stopping set if $S$ is non-empty and for all $c_{j} \in N(S),\left|N\left(\left\{c_{j}\right\}\right) \cap S\right| \geq 2$ i.e., every vertex connected to some vertex in a stopping set must have at least two neighbors in the stopping set. The stopping distance of a Tanner graph is the size of the smallest stopping set in the graph. We define two decision problems concerning stopping sets:

Problem 1: STOPPING SET: Given a Tanner graph $G$ and positive integer $t$, does $G$ have a stopping set of size $t$ ?

Problem 2: STOPPING DISTANCE: Given a Tanner graph $G$ and positive integer $t$, does $G$ have a stopping set of size at most $t$ ?

Note that the corresponding decision problems arising out of the problem of finding the minimum distance of a code were shown to be NP-complete in [15] and [14].

It is clear that if either STOPPING SET or STOPPING DISTANCE can be solved in polynomial time, then invoking the algorithm at most $|L|$ times, the problem of actually finding the stopping distance of a Tanner graph can be solved. Conversely, if there is a polynomial time algorithm for finding the stopping distance of a given Tanner graph $G$, then we can use the algorithm to solve STOPPING DISTANCE since $G$ has stopping distance less than or equal to $t$ if and only if $G$ contains a stopping set of size less than or equal to $t$. Note that it is not immediately clear how to solve STOPPING SET in polynomial time even if a polynomial time algorithm for computing the stopping distance of a Tanner graph is known. 
The notion of NP-completeness was introduced in [11], and is well established in the computer science literature for the analysis of the computational complexity of problems (see [9], [10] for a detailed account). Typically, a problem is posed as a decision problem, i.e., one where the solution consists of answering it with a yes or a no. All inputs for which the answer is a yes form a set. We identify this set with the problem. A decision problem $A$ belongs to the class NP if there exists a polynomial time algorithm $\Pi$ such that, for all $x \in A$, there exists a string $y$ (called a certificate for membership of $x$ in $A$ ), with $|y|$ polynomially bounded in $|x|$, such that $\Pi$ accepts $(x, y)$, whereas, for all $x \notin A, \Pi$ rejects $(x, y)$ for any string $y$ presented to the algorithm. In other words, problems in NP are precisely those for which membership verification is polynomially solvable. We say a decision problem $A$ is polynomial time many-one reducible to a decision problem $B$ if there exists a polynomial time algorithm $\Pi^{\prime}$ such that, given an instance $x$ of $A, \Pi^{\prime}$ produces an instance $z$ of $B$ satisfying $z \in B$ if and only if $x \in A$. In such case, we write $A \preceq_{p} B$. A problem $A \in \mathrm{NP}$ is NP-complete if for every $X \in \mathrm{NP}$, $X \preceq_{p} A$. It is generally believed that NP-complete problems have no polynomial time algorithms.

Given an undirected graph (not necessarily bipartite) $G=$ $(V, E), S \subseteq V$ is a vertex cover in $G$ if for all $(u, v) \in E$ either $u \in S$ or $v \in S$ or both. We will be using in our reductions the following decision problems associated with the computation of vertex covers in a graph.

Problem 3: VERTEX COVER(=): Given a graph $G$ and a positive integer $t$, does $G$ contain a vertex cover of size equal to $t$ ?

Problem 4: VERTEX COVER: Given a graph $G$ and a positive integer $t$, does $G$ contain a vertex cover of size at most $t$ ?

VERTEX COVER is shown to be NP-complete in [10, p. 190]. VERTEX COVER(=) is shown to be NP-complete in [9, pp. 949-950] (in fact, in this reference, the authors refer to this problem as the VERTEX COVER problem).

In the following section we show that both STOPPING DISTANCE and STOPPING SET are NP-complete by establishing polynomial time many-one reductions from VERTEX COVER and VERTEX COVER(=) respectively to the above problems.

\section{HARDNESS OF STOPPING DISTANCE}

Let $(G=(V, E), t)$ be an instance of the VERTEX COVER problem. Let $|V|=n,|E|=m$. Excluding trivial cases of the problem we may assume $1 \leq t \leq n-1$. We shall make the further assumption that $G$ is connected. It is not hard to show that both VERTEX COVER and VERTEX COVER(=) remain NP-complete even when restricted to connected graphs.

The vertex-edge incidence graph of $G$ is the undirected bipartite graph $G^{\prime}=\left(L, R, E^{\prime}\right)$ with $L=V, R=E$ and edges $(e, u)$ and $(e, v)$ in $E^{\prime}$ for each $e=(u, v) \in E$. Fig. 1 shows the vertex-edge incidence graph for a graph $G$ with $n=4$ and $m=3$.

The advantage of assuming that $G$ is connected arises out of the following lemma:
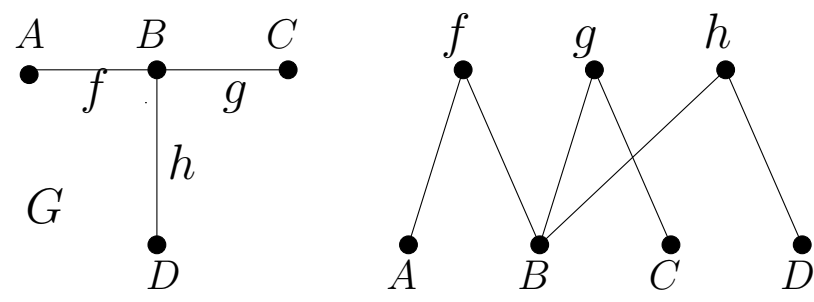

Fig. 1. A graph $G$ and its vertex-edge incidence graph

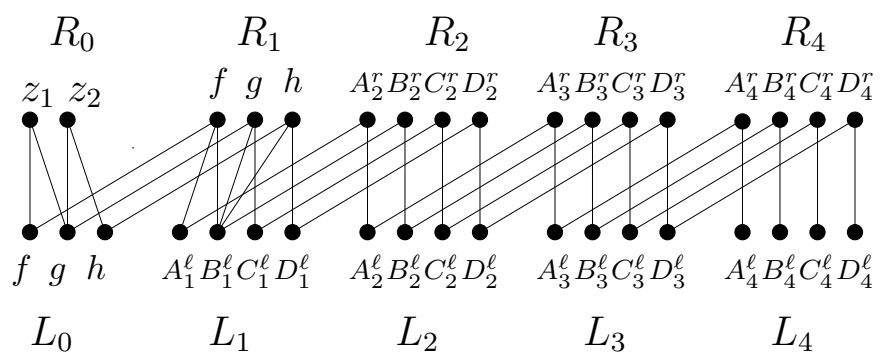

Fig. 2. Construction of $G^{\prime \prime}$

Lemma 1: Let $G^{\prime}=\left(L, R, E^{\prime}\right)$ be the vertex-edge incidence graph of a connected graph $G=(V, E)$. Let $S$ be a stopping set in $G^{\prime}$. Then $S=L$.

Proof: Suppose to the contrary that $L \backslash S \neq \emptyset$. Then, as $G$ is connected there exists $v \in L \backslash S$ and $u \in S$ such that $(u, v) \in E$. Let $e=(u, v)$. Then $e \in N(S)$. Since $S$ is a stopping set $|N(\{e\}) \cap S| \geq 2$. But the only neighbors of $e$ in $G^{\prime}$ are $u$ and $v$. Hence $v \in S$ contradicting $v \in L \backslash S$.

We construct an undirected bipartite graph $G^{\prime \prime}=\left(L, R, E^{\prime \prime}\right)$ as follows: $L=\bigcup_{i=0}^{m+1} L_{i}, R=\bigcup_{j=0}^{m+1} R_{j}$, where, $R_{0}=$ $\left\{z_{1}, \ldots, z_{m-1}\right\}, R_{j}=\left\{u_{j}^{r}, u \in V\right\}$ for $2 \leq j \leq m+1, R_{1}$ and $L_{0}$ are copies of $E$, the edge set of $G$ and $L_{i}=\left\{u_{i}^{\ell}, u \in V\right\}$ for $1 \leq i \leq m+1$. Edges in $G^{\prime \prime}$ are connected as follows:

- Connect $u_{i}^{\ell} \in L_{i}$ to $u_{i}^{r} \in R_{i}, 2 \leq i \leq m+1$.

- Connect $u_{i}^{\ell} \in L_{i}$ to $u_{i+1}^{r} \in R_{i+1}, 1 \leq i \leq m$.

- For each $e=(u, v)$ in $E$, connect $e \in R_{1}$ to $u$ and $v$ in $L_{1}$.

- For each $e \in E$, connect $e \in L_{0}$ to $e \in R_{1}$.

- For the purpose of defining the edges between $R_{0}$ and $L_{0}$, temporarily re-label vertices in $L_{0}$ as $e_{1}, e_{2}, . . e_{m}$ in some arbitrary way. Add the edges $\left(e_{i}, z_{i}\right)$ for $1 \leq i \leq m-1$ and the edges $\left(e_{i}, z_{i-1}\right)$ for $2 \leq i \leq m$.

The example in Fig. 2 illustrates the construction of $G^{\prime \prime}$ for the graph $G$ in Fig. 1. The graph $G^{\prime \prime}$ consists of a copy of the vertex-edge incidence graph of $G$ (vertex sets $L_{1}$ and $\left.R_{1}\right)$. Additionally, there are $m$ copies of the vertex set $V$ on the left $\left(L_{2}, L_{3}, \ldots, L_{m+1}\right)$ and right $\left(R_{2}, R_{3}, \ldots R_{m+1}\right)$. The connections between $R_{0}$ and $L_{0}$ ensure that any stopping set in $G^{\prime \prime}$ containing any one vertex in $L_{0}$ must contain the whole of $L_{0}$. The vertex $u_{i}^{r}$ in $R_{i}$ has neighbors $u_{i-1}^{\ell}$ and $u_{i}^{\ell}$ for each $2 \leq i \leq m+1$ and each $u \in V$. This ensures that if a stopping set $S$ in $G^{\prime \prime}$ contains $u_{i}^{\ell}$ for some $i \in\{1,2, . . m+1\}$ then all the $m+1$ vertices $u_{1}^{\ell}, u_{2}^{\ell}, \ldots ., u_{m+1}^{\ell}$ must be present in $S$. These observations, summarized below, play a crucial role in 
the arguments that follow.

Observation 1: A stopping set $S^{\prime}$ in $G^{\prime \prime}$ satisfies $u_{i}^{\ell} \in S^{\prime}$ for some $1 \leq i \leq m+1$ if and only if it satisfies $u_{i}^{\ell} \in S^{\prime}$ for every $1 \leq i \leq m+1$. Moreover either $L_{0} \subseteq S^{\prime}$ or $L_{0} \cap S^{\prime}=\emptyset$.

The following two claims establish the connection between vertex covers in $G$ and stopping sets in $G^{\prime \prime}$.

Lemma 2: If $G$ contains a vertex cover $S$ of size $t$ for some $1 \leq t \leq n-1$ then $G^{\prime \prime}$ contains a stopping set of size $t(m+$ 1) $+m$.

Proof: Consider the set $S^{\prime}=L_{0} \cup\left\{u_{i}^{\ell}: u \in S, 1 \leq\right.$ $i \leq m+1\}$ in $G^{\prime \prime}$. Clearly $S^{\prime}$ has $t(m+1)+m$ elements. Let $w \in N\left(S^{\prime}\right)$. Then either $w=u_{i}^{r}$ for some $u \in S, i \in$ $\{2,3, \ldots m+1\}$ or $w \in R_{1}$ or $w \in R_{0}$. In the first case, both $u_{i}^{\ell}$ and $u_{i-1}^{\ell}$ are neighbors of $w$. If $w \in R_{1}$, then by construction, $w$ must correspond to some edge $e=(u, v)$ in $E$. Since $L_{0} \subseteq S^{\prime}, e \in L_{0}$ is a neighbor of $w$. Since $S$ is a vertex cover in $G$, either $u$ or $v$ or both must belong to $S$. Hence one or both of $u_{1}^{\ell}$ and $v_{1}^{\ell}$ are neighbors of $w$ which belong to $S^{\prime}$. Finally if $w \in R_{0}$, then both the neighbors of $w$ are in $L_{0}$, and therefore in $S^{\prime}$. Thus in all cases $w$ has at least two neighbors in $S^{\prime}$. Consequently $S^{\prime}$ is a stopping set.

We now prove that every stopping set in $G^{\prime \prime}$ of size less than $n(m+1)$ must correspond to some vertex cover of size $t$ in $G$ for some $1 \leq t \leq n-1$ and must have size exactly $t(m+1)+m$

Lemma 3: Let $S^{\prime}$ be a stopping set in $G^{\prime \prime}$ of size less than $n(m+1)$. Then the following must hold:

- $L_{0} \subseteq S^{\prime}$,

- $\left|S^{\prime}\right|=t(m+1)+m$ for some $1 \leq t \leq n-1$ and $\left|S^{\prime} \cap L_{i}\right|=t$ for every $1 \leq i \leq m+1$

- $S=\left\{u \in V: u_{1}^{\ell} \in S^{\prime}\right\}$ is a vertex cover of size $t$ in $G$. Proof: Suppose $L_{0}$ is not contained in $S^{\prime}$. Then by Observation 1, $L_{0} \cap S^{\prime}=\emptyset$. Since $S^{\prime} \neq \emptyset$, There must be some $u \in V$ and $i \in\{1,2, . ., m+1\}$ such that $u_{i}^{\ell} \in S^{\prime}$. By Observation $1, u_{1}^{\ell} \in S^{\prime}$. Since vertices in the set $R_{1}$ are connected only to $L_{1}$ and $L_{0}$, every neighbor of $S^{\prime}$ in $R_{1}$ must have two neighbors in $S^{\prime} \cap L_{1}$ in order for $S^{\prime}$ to satisfy the conditions of a stopping set. In other words, $S^{\prime} \cap L_{1}$ must be a stopping set in the subgraph of $G^{\prime \prime}$ induced by the vertices $L_{1} \cup R_{1}$. Note that this subgraph is the vertex-edge incidence graph of $G$. Applying Lemma 1 we get $S^{\prime} \cap L_{1}=L_{1}$. Hence Observation 1 shows that $S^{\prime}=\bigcup_{i=1}^{m+1} L_{i}$. But in that case $\left|S^{\prime}\right|=n(m+1)$, a contradiction. Hence $L_{0} \subseteq S^{\prime}$ and $\left|L_{1} \cap S^{\prime}\right|<n$. Let $\left|S^{\prime} \cap L_{1}\right|=t$ for some $1 \leq t \leq n-1$. Applying Observation 1 once again, $\left|S^{\prime} \cap L_{i}\right|=t$ for all $1 \leq i \leq m+1$. Hence $\left|S^{\prime}\right|=t(m+1)+m$.

To complete the proof of the lemma, it is sufficient to prove that $S=\left\{u \in V: u_{1}^{\ell} \in S^{\prime}\right\}$ is a vertex cover of $G$. Since $L_{0} \subseteq S^{\prime}, R_{1} \subseteq N\left(S^{\prime}\right)$. Since every vertex $e$ in $R_{1}$ has only one neighbor in the set $L_{0}$, for $S^{\prime}$ to satisfy the stopping set condition $e$ must have a neighbor in $L_{1} \cap S^{\prime}$. Then, by construction $\left\{u \in V: u_{1}^{\ell} \in S^{\prime}\right\}$ must be a vertex cover in $G$ as required.

As a consequence of Lemma 2 and Lemma 3 we have:

Corollary 1: $G$ has a vertex cover of size $t$ if and only if $G^{\prime \prime}$ has a stopping set of size $t(m+1)+m, 1 \leq t \leq$ $n-1$. Hence $(G, t) \in \operatorname{VERTEX} \operatorname{COVER}(=)$ if and only if $\left(G^{\prime \prime}, t(m+1)+m\right) \in$ STOPPING SET.

Corollary 2: $G$ has a vertex cover of size at most $t$ if and only if $G^{\prime \prime}$ has a stopping set of size at most $t(m+1)+m$, $t \in\{1,2, . ., n-1\}$. Hence $(G, t) \in$ VERTEX COVER if and only if $\left(G^{\prime \prime}, t(m+1)+m\right) \in$ STOPPING DISTANCE.

We are now ready to prove:

Theorem 1: STOPPING DISTANCE and STOPPING SET are NP-complete

Proof:

Since $G^{\prime \prime}$ can be constructed from $G$ in polynomial time $(O(m n)$ time suffices), it follows that VERTEX $\operatorname{COVER}(=)$ $\preceq_{p}$ STOPPING SET and VERTEX COVER $\preceq_{p}$ STOPPING DISTANCE from Corollary 1 and Corollary 2 respectively. It is easy to verify whether a given set of left vertices of a bipartite graph forms a stopping set in time linear in the size of the graph. Hence both STOPPING DISTANCE and STOPPING SET belong to the class NP.

As a consequence, we have:

Corollary 3: There is no polynomial time algorithm for computing the stopping distance of a Tanner graph unless $\mathrm{P}=\mathrm{NP}$.

\section{ACKNOWLEDGMENT}

The authors would like to thank Dr. L. Sunil Chandran for useful discussions, and the anonymous referees for their helpful comments.

\section{REFERENCES}

[1] C. Di, D. Proietti, I. E. Telatar, T. J. Richardson, and R. L. Urbanke, "Finite length analysis of low-density parity-check codes on the binary erasure channel," IEEE Trans. Inform. Theory., vol. 48, no. 6, pp. 15701579, June 2002.

[2] C. Di, A. Montanari and R. Urbanke, "Weight distribution of LDPC code ensembles: Combinatorics meets statistical physics," in Proc. IEEE Int. Symp. Inform. Theory, Chicago, IL., July 2004, p. 102.

[3] A. Orlitsky, K. Viswanathan, and J. Zhang, "Stopping set distribution of LDPC code ensembles," IEEE Trans. Inform. Theory, vol. 51, no. 3, March 2005, pp. 929-953.

[4] T. Tian, C. Jones, J. D. Villasenor, and R. D. Wesel, "Construction of irregular LDPC codes with low error floors," in Proc. IEEE Int. Conf. Comm., Seattle, Washington, May 2003, pp. 3125-3129.

[5] A. Ramamoorthy and R. Wesel, "Construction of short block length irregular LDPC codes," in Proc. IEEE Int. Conf. Comm., Paris, June 2004, pp. 410-414.

[6] A. Orlitsky, R. Urbanke, K. Viswanathan, and J. Zhang, "Stopping sets and girth of Tanner graphs," in Proc. IEEE Int. Symp. Inform. Theory, Lausanne, June 2002, p. 2.

[7] M. Schwartz and A. Vardy, "On the stopping distance and the stopping redundancy of codes," IEEE Trans. Inform. Theory vol. 52, no. 3, pp. 922-932, March 2006.

[8] R. Michael Tanner, "A recursive approach to low-complexity codes," IEEE Trans. Inform. Theory, vol. 27, no. 5 pp. 533-547, Sept 1981.

[9] T. H. Cormen, C. E. Leicerson, and R. L. Rivest, Introduction to Algorithms, MIT Press, 1990.

[10] M. R. Garey and D. S. Johnson, Computers and Intractability: A Guide to the Theory of NP-completeness, W. H. Freeman, 1979.

[11] S. Cook, "The complexity of theorem proving procedures," in Proc. Third ACM Ann. Symposium on Theory of Computing, Shaker Heights, Ohio, May 1971, pp.151-158.

[12] H. Pishro-Nik and F. Fekri, "On decoding of low-density parity-check codes over the binary erasure channel," IEEE Trans. Inform. Theory, vol. 50, no. 3, pp. 439-454, March 2004.

[13] J. Han and P. Siegel, "Improved upper bounds on stopping redundancy," preprint available at: http://www.arXiv.org, cs.IT/0511056. 
[14] A. Vardy, "The intractability of computing the minimum distance of a code," IEEE Trans. Inform. Theory, vol. 46 no. 6 pp. 1757-1766, Nov. 1997.

[15] E. R. Berlekamp, R. J. McEliece, and H. C. A van Tilborg, "On the inherent intractability of certain coding problems," IEEE Trans. Inform. Theory, vol. 24 no. 3, pp. 384-386, May 1978.

Priti Shankar received the B. Tech in Electrical Engineering at the Indian Institute of Technology, Delhi in 1968, and the Ph.D. degree from the University of Maryland, College Park in 1972. Since 1973, she has been at the Department of Computer Science and Automation, Indian Institute of Science, Bangalore, where she is currently Professor. Her interests are in theoretical computer science, compiler tools and coding theory. Dr. Shankar is a member of the ACM and the American Mathematical Society.

Karunakaran Murali Krishnan received the M.Tech degree in Computer Science and Engineering from the Indian Institute of Technology, Kanpur in 1998. He is affiliated to the National Institute of Technology Calicut since 1998, where he is a Lecturer in Computer Engineering. He is currently a PhD. student at the Indian Institute of Science, Bangalore. His research interests are in coding theory and computational complexity. 